\title{
Decreased health related quality of life among Hispanic breast cancer survivors
}

\begin{abstract}
Introduction: The breast cancer survivors' population is growing due to the increased rate of early diagnosis and appropriate management. Hispanics (Latinos) constitute a rapidly growing population. Hispanic cancer survivors are often left without appropriate follow up care after completion of therapy, partly because of the lack of financial support and resources, and limited awareness of possible subsequent physical and mental health problems among those survivors. We sought to evaluate the quality of life (QoL) of the Hispanic breast cancer survivors in El Paso, TX, a large American-Mexican border city, using a validated Health related-Quality of life survey (HRQOL SF-36)
\end{abstract}

Materials and methods: After IRB approval, we recruited Hispanic women within their first 5years post-diagnosis with Stages I, II, or III breast cancer, and who have completed their active chemotherapy and/or radiation therapy. They were provided with a written SF-36 questionnaire. We analyzed the two main components of the SF36 , the physical health through a Physical Component Summary (PCS), and the mental health, through a Mental Component Summary (MCS). Results were compared with the US healthy population (mean 50) using a one sample t-test. SF-36 scores were also compared with similar studies using a two sample t-test.

Results: The study suggests that both the physical and mental components of QoL were low. PCS was significantly lower than one standard deviation below the US norm whereas MCS was approximately one third of a standard deviation below the US norm; $47 \%$ of all survivors reported that physical or emotional problems interfere with their normal social activities; $39 \%$ of all patients reported having emotional problems like depression and anxiety; and $77 \%$ indicated difficulty performing their work. The mean PCS and MCS scores of our Hispanic population were inferior to similar nonHispanic breast cancer survivors' population in other studies.

Conclusion: Hispanic breast cancer survivors in El Paso, TX have decreased mental and physical health related QoL compared to healthy U.S. women and possibly other breast cancer survivors in the U.S. Further investigation is needed to determine the possible underlying causes of health disparities in breast cancer survivors and identify strategies to improve these outcomes.

Keywords: breast cancer, Hispanic, survivors, quality of life
Volume 2 Issue I - 2016

\section{Zeina A Nahleh, ' Alok Dwivedi, ${ }^{2}$ Tony Khang, ${ }^{3}$ Aleem Sattar, ${ }^{3}$ Arafat $\mathrm{H}$ Tfayli, ${ }^{4}$ Safa} Farrag' Indika Mallawaarachchi ${ }^{5}$

'Department of Internal Medicine, Texas Tech University Health Sciences Center (TTUHSC), USA

${ }^{2}$ Department of Biomedical Sciences, Texas Tech University Health Sciences Center (TTUHSC), USA

${ }^{3}$ Paul Foster School of Medicine, Texas Tech University Health Sciences Center (TTUHSC), USA

${ }^{4}$ Department of Internal Medicine, American University of Beirut Medical Center, Lebanon

${ }^{5}$ Division of Biostatistics, Epidemiology, Department of Biomedical Sciences, USA

Correspondence: Zeina Nahleh, Division of Hematology-

Oncology, Department of Internal Medicine, Texas Tech

University Health Sciences Center (TTUHSC), El Paso

TX-79905, USA, Tel 9|5-2।5-5। 95, Fax 9|5-545-6634,

Email zeina.nahleh@ttuhsc.edu; Zeina.Nahleh@ttuhsc.edu

Received: December 12, 2015 | Published: January 28, 2016
Abbreviations: UBCC, university breast care center; TTUHSC, texas tech university health science center; QOL, quality of life; sf: short-form; PF, physical functioning; RP, role function-physical; BP, bodily pain; SF, social functioning; $\mathrm{MH}$, mental health RE, role function-emotional; VT, vitality; GH, general health; PCS, physical component summary; MCS, mental component summary; IQR, inter quartile range; SD, standard deviation; SERMs, selective estrogen receptors modulators

\section{Introduction}

The number of cancer survivors is growing for several reasons, including early detection, more accurate diagnosis, and more effective treatment, currently exceeding 13.7 million in the United States. ${ }^{1}$ The number of breast cancer survivors currently exceeds 2.5 million and comprises the largest proportion of all survivors. ${ }^{2}$ However, at least one third of survivors experience ongoing physical, psychological or financial consequences of their cancer diagnosis and treatment. ${ }^{3,4}$ Appropriate follow-up care is often not delivered and the psychosocial needs of cancer patients are often not addressed. Also, many patients complete their primary treatment for cancer unaware of their heightened health risks and are ill prepared to manage their future health care needs. ${ }^{4}$ As a result, the oncology community and many national organizations have begun to focus on survivorship as a central component of oncology care..$^{3-5}$ Cancer survivorship is a relatively new phenomenon, so the current pace of research and development of effective models of care still lags behind the need. Hispanic/Latinas breast cancer survivors may have additional needs compared to breast cancer survivors from other ethnic groups.

An exploratory research at the University of Chicago including 989 newly diagnosed breast cancer patients suggested an association between psychosocial stress post-diagnosis (in the form of fear, anxiety, or isolation) and breast cancer aggressiveness. The rate of post-diagnosis psychosocial stress was about two-fold higher in Hispanics. ${ }^{6}$ Another study including 117 Hispanic breast cancer survivors at the University of Texas, San Antonio, TX found that Hispanic breast cancer survivors have a high rate of depression and that, piled on other barriers like cost factors and underinsurance, prevents many of them from getting screenings for other cancers. ${ }^{7}$ 
Also, an analysis of twenty-two studies evaluating QoL in Hispanic (Latina) women with breast cancer compared to other racial/ethnic groups concluded that Latinas appear to be at risk for poor QoL following a breast cancer diagnosis relative to non-Latinas. Relatively little is known about the mechanisms that explain these health-related discrepancies. ${ }^{8}$ The purpose of this study was to evaluate the QoL of the Hispanic breast cancer survivors in El Paso, TX using a validated Health related survey SF-36,10 or its Spanish version. ${ }^{11}$ The study population represents a relatively homogenous group of patients in the Mexican-US border city of El Paso. Understanding specific needs and ethnic differences in QoL among breast cancer survivors can inform future interventions targeted at improving health status for this patient population.

\section{Materials and methods}

After obtaining approval from the Institutional Review Board, we recruited consecutive self-identified-Hispanic patients from the University Breast Care Center (UBCC) at Texas Tech University Health Science Center (TTUHSC) in El Paso over a 6month period (February through July 2012) in a cross sectional study design. The study eligibility criteria required that Hispanic women fall within the first 5years post-diagnosis with Stages I, II, or III breast cancer, have completed their active chemotherapy and/or radiation therapy, and that they be disease free. Patients were provided with written SF-36 questionnaire to assess their health-related QoL. This is a validated multi-purpose, short-form health survey with 36 questions..$^{9,10} \mathrm{~A}$ Spanish version was given to Spanish speaking patients. ${ }^{11}$ The SF36 is composed of 8 multi-item scales (36 items) assessing physical function (10 items), role limitations due to physical health problems (4 items), bodily pain (2 items), general health (5 items), vitality (4 items), social functioning ( 2 items), role limitations due to emotional problems ( 3 items) and emotional well-being (5 items). The $36^{\text {th }}$ item, which asks about health change, is not included in the scale or summary scores. These eight scales can be aggregated into two summary measures: the Physical (PCS) and Mental (MCS) Component Summary scores. ${ }^{12,13}$ PCS and MCS are simply weighted aggregations of scores for the eight SF36 subscales, to simplify the analysis. The PCS is related to physical functioning, role physical, bodily pain and general health dimensions and the MCS to vitality, social functioning, role emotional and mental health. The SF-36 summary scores (PCS36 and MCS-36) range from 0 to 100 , with higher scores representing better self-reported health, and they are calculated using standard (US-derived) scoring algorithms from Ware et al. ${ }^{12}$

General health and vitality are domains shared by PCS and MCS. In addition, PCS encompasses physical functioning, role-physical, and bodily pain, whereas MCS includes social functioning, role-emotional, and mental health. PCS and MCS are presented as T-scores. In each of the 8 scale scores, the missing are computed with the corresponding average score and considered to be a complete score if half or more items are not missing. ${ }^{14}$ Quantitative variables were summarized using mean, standard deviation (SD), median, interquartile range (IQR) and range as appropriate. The categorical variables were presented using frequency and proportions. The PCS and MCS were compared with the US normal healthy population (mean 50) using a one sample t-test. SF-36 scores were also compared with similar studies reported in the literature using a two sample t-test. PCS and MCS were also compared in this study according to age category $(<50, \geq 50$ years), duration since diagnosis ( $\leq 3,>3$ years), stage (I, II and III), received chemotherapy (no and yes), and received hormonal therapy (no and yes) using an unpaired t-test or a one way ANOVA. P-values less than 0.05 were regarded as significant results. Statistical analysis was done using SAS 9.3. Bar diagrams were used to delineate important findings in the study. Error in bars represents standard deviation.

\section{Results and discussion}

A total of 102 eligible Hispanic breast cancer survivors with stages I-III breast cancer within 5Syears of diagnosis were recruited. The mean age was 57years (range 38-84). Patient characteristics are summarized in Table 1 . The majority of the patients were recruited within their first 3years of diagnosis; $67.65 \%$ of the patients received chemotherapy, and $60.4 \%$ patients received hormonal therapy. Most of the patients were Spanish language speakers. A summary of scale scores is provided in Table 2. The participants had lower scores on emotional role function, general health and physical functioning followed by average mental health and social functioning scales. The mean PCS representing the mean for the SF-36 Physical Health was 40.4 and the mean MCS for Mental Health was 47.8. This indicates that PCS of study participants was significantly less than one standard deviation below the US norm whereas MCS was approximately one third of a standard deviation below the US norm (Figure 1). Remarkably, $47 \%$ of all survivors reported that physical or emotional problems interfere with their normal social activities; 39\% of all patients reported having emotional problems at work or other daily activities such as feeling depressed or anxious; and significantly $77 \%$ of the study population indicated difficulty performing their work. No significant correlation between PCS and MCS was obtained for our survivors $(\mathrm{r}=0.12, \mathrm{p}=0.20)$. The comparison of mean scores of PCS and MCS for our Hispanic breast cancer survivors with similar scores from other studies of breast cancer survivors reported in the literature is shown in Figure 2. Our survivors scored less on PCS (40.41 versus 50.2, $\mathrm{P}<0.0001)$ and MCS (47.82 versus $49.4 \mathrm{p}=0.163)$ as compared to Ganz et al. ${ }^{15}$ Also, PCS (40.41 versus 50.9, $\mathrm{P}<0.0001$ ) and MCS (47.82 versus $50.0 \mathrm{P}=0.052$ ) were found to be inferior as compared to same scores reported in Kendall et al. ${ }^{16}$ The study participants scored less on PCS (40.41 versus 46.1, $\mathrm{P}<0.0001$ ) but not significantly different on MCS (47.82 versus 49.6, $\mathrm{p}=0.204$ ) as compared to Wilson et al. ${ }^{17}$

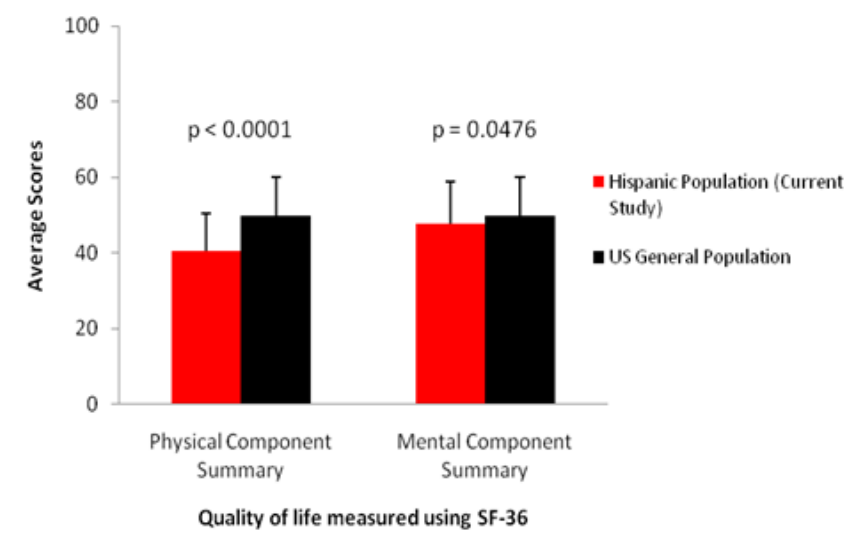

Figure I Comparison of physical and mental components of SF-36 of Hispanic breast cancer survivors with US healthy normal population.

In summary, the mean scores were inferior for both PCS and MCS in our patient population. The differences in the means of PCS and MCS were not found to be statistically significant according to age, duration of diagnosis, stage of cancer or whether chemotherapy was 
received (Table 3). However, the comparison of SF-36 subscales reveals that physical body pain was significantly higher for patients who received chemotherapy than those who did not. The difference in the means for PCS was not found to be statistically significantly different according to hormonal therapy status. However, notably, patients receiving hormonal therapy had lower MCS (45.48 versus $51.5, \mathrm{p}=0.006)($ Table 3$)$.

Table I Characteristics of the participants in the QoL survey

\begin{tabular}{ll}
\hline Variable & $\mathbf{N}(\%)$ \\
\hline Age [mean(SD) years] & $57.75(I 0.03)$ \\
Duration since diagnosis [median(IQR) years] & $2.00(I, 4)$ \\
Stage & \\
I & $33(32.35)$ \\
II & $47(46.08)$ \\
III & $22(21.57)$ \\
Chemotherapy Received & \\
Yes & $69(67.65)$ \\
No & $33(32.35)$ \\
Hormonal Therapy Received & \\
Yes & $61(60.40)$ \\
No & $40(39.60)$ \\
Language & \\
English & $23(22.55)$ \\
Spanish & $79(77.45)$ \\
\hline
\end{tabular}

QoL, quality of life; SD, standard deviation; IQR, interquartile range

Table 2 Summary of SF-36 QoL scale scores

\begin{tabular}{llll}
\hline Variable & Mean & SD & Median \\
\hline Physical Functioning & 60.65 & 26.77 & 65 \\
Role Function-Physical & 53.75 & 45 & 50 \\
Bodily Pain & 55.35 & 24.47 & 52 \\
Social Functioning & 69.07 & 27.04 & 75 \\
Mental Health & 70.5 & 19.27 & 68 \\
Role Function-Emotional & 61.74 & 43.09 & 100 \\
Vitality & 50.68 & 19.53 & 50 \\
General Health & 61.3 & 20.43 & 62 \\
\hline
\end{tabular}

SF, social functioning; QoL, quality of life; SD, standard deviation

This study using a cross-sectional design and standardized QoL measure (SF-36) provides new information about the QoL in the shortterm (within 5years post diagnosis) of disease-free, breast cancer survivors in a relatively ethnically homogenous Hispanic population on the American -Mexican Border in El Paso, TX. This study suggests that Hispanic breast cancer survivors have decreased mental and physical health related QoL compared to the population norm for healthy U.S women and possibly other breast cancer survivors in the U.S. In another study by Ganz et al., ${ }^{15} 577$ survivors with stage I and II breast cancer were surveyed using SF 36 questionnaire at a mean 6years of diagnosis, Mean age of 50years, including $83 \%$ whites and $8.9 \%$ black, $62 \%$ having received chemotherapy. In comparison, the Hispanic breast cancer survivors in our study with clinical similarities in regards to age, stage, and duration since diagnosis scored less on PCS and MCS. In comparison to another survivor population reported by Kendall et al., ${ }^{16}$ surveying 371 breast cancer survivors, all whites, mean age 50 and at a mean 13.2 years after diagnosis, $45 \%$ having had chemotherapy, the survivors in El Paso, TX again scored significantly less on both PCS and MCS. Similarly, the survivors in El Paso, TX had lower PCS as compared to another study with a similar design by Wilson et al. ${ }^{17} \mathrm{~A}$ case control study by Pinheiro et al., ${ }^{18}$ to assess the changes in health related quality of life using the SF-36 form, comparing non-Hispanic whites with Hispanics, African Americans, and Asians found that before cancer diagnosis, non-Hispanic whites had better health related quality of life scores than African Americans and Hispanics on the Role-Physical and Role-Emotional SF-36 subscales. However, they also reported that cancer diagnosis and treatment negatively impacted individuals' lives regardless of race or ethnicity. However, gaps were found between racial and ethnic groups (compared with controls) before and after cancer diagnosis for some SF-36 health related quality of life measures. In our study, both physical and mental health scores are lower as noted above, due possibly to lower baseline quality of life measures before diagnosis. After diagnosis and treatment, it appears that both mental and physical health measures are low but physical health is especially significantly affected. No major differences were seen based on age, interval since diagnosis, and chemotherapy. However, studies with a longer follow up (more than 5years after initial diagnosis) have suggested that survivors with no past systemic adjuvant chemotherapy have higher levels of functioning long term after primary treatment. ${ }^{19}$ However this study is in agreement with other reports suggesting increased post-diagnosis psychosocial stress in Hispanics. ${ }^{6,8}$

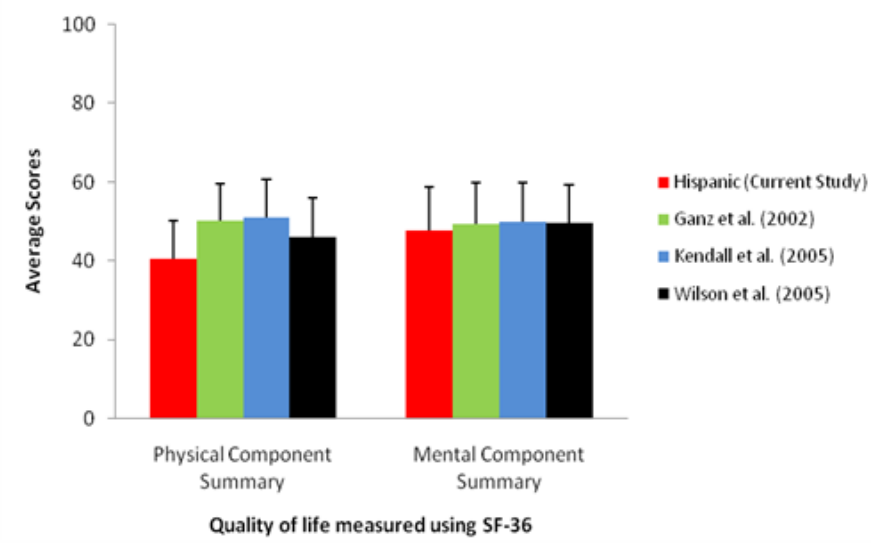

Figure 2 Comparison of physical and mental components of SF-36 of Hispanic breast cancer survivors with other breast cancer survivor's studies.

A possible biological mechanism for the increased stress is that perceived stress affect the glucocorticoid pathway. Also, social/ environmental stress could affect epigenetics, such as DNA methylation, and increased stress can impact immune function adversely. ${ }^{6}$ Also, the differences in the means for PCS was not found to be statistically significantly different according to whether participants are receiving or have received hormonal therapy (compared to those who did not). However, notably patients receiving hormonal therapy had lower MCS. This finding is interesting since one would expect that physically- related side effects of aromatase inhibitors like 
arthralgias, arthritis, and joint stiffness would make a more significant difference and affect more bodily and physical functioning compared to participants not receiving hormonal therapy, this was not the case in our study. However, hormonal therapy (majority received aromatase inhibitors), was found to have a negative effect on mental health and cognitive function. This is consistent with several trials studying the impact of hormonal therapy on cognitive function and brain metabolism. ${ }^{20-23}$ While, the evidence suggests mostly that therapy with Selective Estrogen Receptors Modulators (SERMs) such as tamoxifen does not have a negative impact on the cognitive functioning of women, ${ }^{20}$ studies focusing on aromatase inhibitor therapy have yielded somewhat mixed results. ${ }^{22,23}$ A pilot study evaluated cognitive function in a small subset of women enrolled for at least 3years on the Anastrozole, Tamoxifen Alone or Combined (ATAC) trial, ${ }^{22}$ who completed a battery of neuropsychologic tests found that the patients were impaired on a processing speed task $(\mathrm{p}=0.032)$ and on a measure of immediate verbal memory $(\mathrm{p}=0.026)$. The results showed specific impairments in processing speed and verbal memory. Verbal memory may be especially sensitive to changes in estrogen levels; a finding commonly reported in studies of hormone replacement therapy in healthy women. ${ }^{22}$ The International Breast Cancer Intervention Study II (IBIS II) evaluated the effect of anastrozole versus placebo in 6,000 postmenopausal women at increased risk for breast cancer. Cognitive testing was performed at baseline, 6months, and 2 and 5years (study completion) and showed little or no impairment of cognitive performance with the use of anastrozole compared with placebo in postmenopausal women at high risk of developing breast cancer. ${ }^{23}$

Our breast cancer population is unique, not only due to the predominance of Hispanics with possible heightened social stresses and low socioeconomic status, but also due to the fact that many of the Hispanic breast cancer patients in El Paso, TX are diagnosed at a younger age, $32 \%$ being younger than 50years at diagnosis. Although we did not see major differences in QoL based on age, possibly due to small sample size, other studies have suggested that standardized measures of QoL identify worse outcomes and more frequent depressive symptoms in breast cancer survivors aged 50years or younger when compared with the general age-matched population of women without cancer and to older women (aged $>50$ years) with breast cancer. ${ }^{24}$

Table 3 Comparison of physical component summary(PCS) $S$ and mental component summary(MCS)

\begin{tabular}{|c|c|c|c|c|c|c|c|}
\hline & $\mathbf{N}$ & PCS mean & SD & P-Value & MCS mean & SD & P-value \\
\hline Age & & & & 0.786 & & & 0.989 \\
\hline$<50$ years & 21 & 39.84 & 10.91 & & 47.79 & 9.9 & \\
\hline$\geq 50$ years & 81 & 40.56 & 9.73 & & 47.83 & 11.3 & \\
\hline Duration of Diagnosis & & & & 0.686 & & & 0.797 \\
\hline$\leq 3$ years & 75 & 40.16 & 9.66 & & 47.65 & 10.86 & \\
\hline$>3$ years & 27 & 41.12 & 10.82 & & 48.3 & 11.52 & \\
\hline Stage & & & & 0.409 & & & 0.669 \\
\hline I & 33 & 39.26 & 10.99 & & 46.4 & 10.84 & \\
\hline II & 47 & 41.84 & 9.74 & & 48.51 & 10.87 & \\
\hline III & 22 & 39.09 & 8.59 & & 48.46 & 11.72 & \\
\hline Chemotherapy Received & & & & 0.359 & & & 0.173 \\
\hline Yes & 69 & 41.07 & 9.57 & & 48.93 & 10.18 & \\
\hline No & 33 & 39.05 & 10.67 & & 45.51 & 12.35 & \\
\hline Hormonal Therapy Received & & & & 0.76 & & & 0.006 \\
\hline Yes & 61 & 40.27 & 9.66 & & 45.48 & 11.11 & \\
\hline No & 40 & 40.9 & 10.43 & & 51.51 & 9.96 & \\
\hline
\end{tabular}

PCS, physical component summary; MCS, mental component summary; SD, standard deviation

\section{Conclusion}

This study suggests that Hispanic breast cancer survivors in El Paso, TX have decreased mental and physical health related QoL compared to the population norm for healthy U.S women and possibly other breast cancer survivors in the U.S. Survivorship care programs specifically designed to address their unmet needs should be implemented. These programs should take into consideration cultural variables and should focus on specific physical improvement and stress-reduction strategies as well as improvement of cognitive function, psychological well-being and functional wellness. ${ }^{25,26}$
Barriers that patients face in receiving appropriate survivorship care should also be addressed including a fragmented and poorly coordinated health care system, an absence of a focus of responsibility for follow-up care, and a lack of guidance on how cancer survivors can maximize their own health outcomes. Barriers that health care providers face in delivering care should also be considered including lack of a delivery system supports that would allow them to overcome some of the obstacles posed by fragmented cancer care, and lack of adequate reimbursement of many services in survivor care. ${ }^{3}$ Specific barriers that Hispanic cancer survivors face should be addressed including underinsurance, language barriers and cultural beliefs. 


\section{Acknowledgements}

Cancer Prevention and Research Institute of Texas (CPRIT)RP120528.

\section{Conflict of interest}

The author declares no conflict of interest.

\section{References}

1. National cancer institute. Cancer Treatment and Survivorship. Facts and Figures 2012-2013. USA: American Cancer Society; 2012. p. 1-44.

2. Centers for disease control and prevention \& lance armstrong foundation. A National Action Plan for Cancer Survivorship. Advancing public health strategies, Georgia: CDC \& Lance Armstrong Foundation; 2004. $88 \mathrm{p}$.

3. Hewitt M, Greenfield S, Stovall E. From patient to cancer survivor: lost in transition. Washington, USA: National Academics Press; 2006. $534 \mathrm{p}$.

4. McCabe MS, Bhatia S, Oeffinger KC, et al. American society of clinical oncology statement: achieving high-quality cancer survivorship care. $J$ Clin Oncol. 2013;31(5):631-640.

5. Rauscher, G, AL-Alem U, Richard BW. Does psychosocial stress play a role in the etiology of aggressive breast cancer? A cross-sectional study. Cancer Epidemiol Biomarkers Prev. 2011;20:A91.

6. Ramirez A. Depression affected preventive health screening among Latina breast cancer survivors. Washington, USA: American association for cancer research (AACR) science of cancer health disparities; 2011. 849 p.

7. Yanez B, Thompson EH, Stanton AL. Quality of life among Latina breast cancer patients: a systematic review of the literature. J Cancer Surviv. 2011;5(2):191-207.

8. Hays RD, Sherbourne CD, Mazel RM. The RAND 36- item health survey 1.0. Health Econ. 1993;2(3):217-227.

9. Brazier JE, Harper R, Jones NM, et al. Validating the SF-36 health survey questionnaire: new outcome measure for primary care. $B M J$. 1992;305(6846):160-164

10. Vilagut G, Ferrer M, Rajmil L, et al. The Spanish version of the short form 36 health survey: a decade of experience and new developments. Gac Sanit. 2005;19(2):135-150.

11. Ware JE Jr, Sherbourne CD. The MOS 36-Item short-form health survey (SF-36). I. Conceptual framework and item selection. Medical Care. 1992;30(6):473-483.

12. Ware JE Jr, Kosinski M, Keller. SD. SF-36 Physical and Mental Health Summary Scales: a user's manual. Boston, USA: The Health Institute; 1994.
13. Medical Outcomes Trust: how to score the SF-36 short-form health survey. Medical Outcome Survey. Boston, USA; 1993.

14. Ganz PA, Greendale GA, Petersen L, et al. Breast cancer in younger women: reproductive and late health effects of treatment. J Clin Oncol. 2003;21(22):4184-4193

15. Kendall AR, Mahue-Giangreco M, Carpenter CL, et al. Influence of exercise activity on quality of life in long-term breast cancer survivors. Qual Life Res. 2005;14(2):361-371.

16. Wilson RW, Hutson LM, Vanstry D. Comparison of 2 quality-of-life questionnaires in women treated for breast cancer: the RAND 36Item health survey and the functional living index-cancer. Phys Ther. 2005;85(9):851-860.

17. Ganz PA, Desmond KA, Leedham B, et al. Quality of life in long-term, disease-free survivors of breast cancer: a follow-up study. J Natl Cancer Inst. 2002;94(1):39-49.

18. Pinheiro LC, Wheeler SB, Chen RC, et al. The effects of cancer and racial disparities in health-related quality of life among older Americans: a case-control, population-based study. Cancer. 2015;121(8):1312-1320.

19. Nahleh Z, Zhou J, Ulloa A. Clinicopathologic features of breast cancer in the Texas-Mexico border city of El Paso. USA: ASCO University; 2012.

20. Rugo HS, Ahles T. The impact of adjuvant therapy for breast cancer on cognitive function: current evidence and directions for research. Seminars in Oncology. 2003;30(6):749-762.

21. LeBlanc ES, Janowsky J, Chan BK, et al. Hormone replacement therapy and cognition: sytematic review and meta-analysis. JAMA. 2001;285(11):1489-1499.

22. Jenkins V, Shilling V, Fallowfield L, et al. Does hormone therapy for the treatment of breast cancer have a detrimental effect on memory and cognition? A pilot study. Psychooncology. 2004;13(1):61-66.

23. Jenkins VA, Ambroisine LM, Atkins L, et al. Effects of anastrozole on cognitive performance in postmenopausal women: a randomized, double-blind chemoprevention trial (IBIS II). Lancet Oncol. 2008;9(10):953-961.

24. Howard-Anderson J, Ganz PA, Bower JE, et al. Quality of life, fertility concerns, and behavioral health outcomes in younger breast cancer survivors: a systematic review. J Natl Cancer Inst. 2012;104(5):386-405.

25. Cimprich B, Janz NK, Northouse L, et al. Taking charge: A self-management program for women following breast cancer treatment. Psychooncology. 2005;14(9):704-717.

26. Epplein M, Zheng Y, Zheng W, et al. Quality of life after breast cancer diagnosis and survival. J Clin Oncol. 2011;29(4):406-412. 